\title{
Guest Editorial \\ Introduction to the Special Section on Computer Vision for Intravascular and Intracardiac Imaging
}

$\mathbf{T}$ HIS SPECIAL section of the IEEE TRANSACTIONS ON INFORMATION TECHNOLOGY IN BIOMEDICINE is devoted to advances in computational techniques in the field of intravascular and intracardiac imaging. Despite improvements in a number of noninvasive techniques, cardiac catheterization remains a key clinical tool for assessing the anatomy and physiology of the heart and its associated vasculature. A small catheter (usually a hollow plastic tube with $2-3 \mathrm{~mm}$ diameter) is inserted into a patient's peripheral artery or vein and advanced to the heart. Once properly positioned, it can be used for cross-sectional imaging through a transducer placed in the catheter, ablation of pathological tissue, pressure measurements, or injection of a contrast agent. Diagnostic cardiac catheterization helps in characterization of the extent and severity of cardiac and cardiovascular diseases, and thereby helps in planning for appropriate medical, surgical, or catheter-based treatment. While the majority of patients with coronary artery disease or valvular disease can be tracked with only clinical and noninvasive data, cardiac catheterization and angiographic procedures remain the preferred choice for the physicians for diagnostic or interventional purposes, or both. This special section focuses on the advances for computational methods based on computer vision and image processing principles for intravascular and intracardiac imaging and associated computer-aided diagnosis, treatment, and prognosis.

The motivation for this special section arose from the fact that although there is considerable activity in this relatively new field, there were not many forums or venues where the researchers could come together. Therefore, first, the Guest Editors of this issue organized a workshop at the Medical Image Computing and Computer Assisted Intervention (MICCAI) 2006, in Copenhagen, Denmark. This meeting brought together some pioneers in the field with keynote talks from Prof. Milan Sonka of the University of Iowa, and Dr. Stéphane Carlier of the Cardiovascular Research Foundation (now with Cordis Corporation, Belgium). With the keen interest and participation from the scientists in this community to the workshop, the Guest Editors wanted to carry this momentum to this issue.

Cardiac catheterization can be guided and imaged through various imaging modalities: X-ray, intravascular ultrasound (IVUS), magnetic resonance imaging (MRI), and optical coherence tomography (OCT), in which progressive improvements are poised to have dramatic impact on the diagnosis, analysis, modeling, and treatment of vascular diseases and cardiac disorders. A major disease of interest is atherosclerosis, which is

Digital Object Identifier 10.1109/TITB.2008.920458 characterized by a deposit of plaque in an arterial wall over time. Inflamed, active, and growing atherosclerotic plaques are believed to be "vulnerable" plaques. The disruption of such plaques is considered to be the most frequent cause of heart attack and sudden cardiac death. However, the mechanisms responsible for the sudden conversion of a rupture-prone plaque to a life threatening atherothrombotic lesion are not fully understood yet.

IVUS is a widely used clinical tool in the catheterization laboratory for guiding percutaneous interventions. Recent methods based on spectral analysis of the backscattered radiofrequency (RF) signal offer characterization of plaque into fibrotic/fibrofatty/lipid necrotic core/calcium. OCT is a newer intracoronary imaging modality that provides real-time very high-resolution tomographic visualization of coronary arteries (resolution $\sim 15-20 \mu \mathrm{m}$ ) [1]. Identification of plaque composition, particularly characterization of rupture-prone plaques, from these image modalities is an active research problem in the intravascular image analysis domain [2], [3].

Catheter-based X-ray angiography, which is radiography of blood vessels through the injection of an iodine-containing fluid into the arteries or veins followed by a subtraction image operation, is the current standard for diagnosis of coronary artery disease and in guiding cardiac and peripheral vascular interventions. Other common uses are stenosis quantification, detection of aneurysms, and dilations of the vessels through vascular wall segmentation or edge detection. It is also used to reconstruct a 3-D model of the artery from single-plane, biplane, or very recently rotational angiography to study hemodynamics, stress/strain distribution, and mechanical properties of the arterial wall. The latter application is preferably coupled with IVUS catheterization for an improved understanding of the arterial tissue properties [4], [5].

Catheter ablation involves the selective destruction of the cardiac tissue responsible for arrhythmias via percutaneously placed catheters. Recently, studies for use of late-contrastenhanced MRI (CE-MRI) in such procedures enabled visualization of myocardial scar tissue that contains arrhythmia substrate. Delineation of scar tissue, which could be facilitated by myocardial segmentation, would be useful in clinical studies of myocardial scar and its impact on cardiac disorders.

Echocardiography is a diagnostic imaging technique that employs ultrasound to image organ anatomy. For contrast-enhanced ultrasound, gas-filled microbubbles are injected into the venous system to improve tissue and blood delineation (e.g., evaluating myocardial perfusion). The latest ultrasound systems now employ 3-D real-time imaging. 
Computational and mathematical techniques designed to analyze intravascular images for anatomic modeling as for the 3-D reconstruction of the arterial wall anatomy from X-ray angiography and IVUS, visualization, arterial wall tissue classification particularly from IVUS and OCT, and the assessment of interventional procedures (X-ray and others), cardiac and intracardiac anatomic modeling for myocardium and left ventricle wall, and pathologic tissue (like scar tissue within) are therefore playing a role of increased importance and are currently receiving significant interest [1]-[17].

The Guest Editors invited papers presenting new techniques for any aspect of computational intravascular or intracardiac image analysis and processing. We opted to select a representative set of papers from among a number of very good submissions, which we hope will give readers a reasonable sense for some of the new and exciting research in this field. The research included in this issue covers a wide range of topics, from image-based frame gating of IVUS images, strain and shear analysis using elastograms for studying plaques, new computational methods for extraction of both inner and outer arterial walls from IVUS pullbacks, analysis of plaque characterization methods through feature extraction and appropriate statistical classifiers using IVUS RF data, myocardial and left ventricle segmentation from CE-MRI data in the presence of scar tissue for patients with atrial fibrillation, to 3-D IVUS transducer tracking from single-plane angiography for the purpose of 3-D arterial model reconstruction.

To give a more detailed introduction of the contents of this issue, Baldewsing et al. provides an automated method for reconstructing the elasticity distribution of heterogeneous atherosclerotic coronary plaques. This paper extends previous IVUS modulography work by including a novel divideand-conquer computational strategy for reconstructing arbitrary plaques with a varied compositional structure. This approach is capable of estimating the total burden of dangerous soft thrombogenic material [8].

Maurice et al. presents for the first time, in vivo radial strain and shear along with tangential strain elastograms for both animal and human studies. The method relies on the Lagrangian speckle model estimator to estimate radial/axial as well as circumferential/lateral displacement fields, and shows much promise in characterizing atherosclerotic plaques and mural thrombi [9].

O'Malley et al. address the problem of eliminating motion artifacts in IVUS pullback sequences using image-based frame gating as an alternative to ECG gating. The computationally inexpensive frame gating method automatically chooses the fraction of the cycle that produces the most stable (in terms of interframe similarity) gated set of images [10].

In Jourdain et al., the authors address the problem of correct geometric estimation of the 3-D model of the artery from a single angiogram. For this purpose, they present a method for IVUS transducer localization through a single-plane X-ray image sequence, given the pullback speed of the IVUS transducer as prior information as well as a rough estimate of the starting depth or mean depth of the trajectory of the transducer in the scanned artery using both full- and weak-perspective camera models for the X-ray system [11].

Katouzian et al. address the challenges of in vivo atherosclerotic plaque characterization in IVUS imaging, which is reflected by changes in spectral contents of the ultrasound RF signal. They analyzed a number of spectral features (e.g., spectral slope and intercept) that were used before and shown to be prone to variations of catheter and tissue spectral parameters. The authors made a rigorous attempt to address two important questions in tissue characterization: how to extract the most informative features; and which classification algorithm is most appropriate to the problem? [12].

Folkesson et al. present an automatic segmentation method for myocardium with scar tissue from contrast-enhanced MRI data for the final purpose of aiding the catheter ablation therapy in atrial fibrillation disorder. They incorporated a supervised learning in terms of a statistical classifier into a geodesic active region framework in order to particularly deal with irregular appearance due to scar tissue in the myocardium. A shape prior obtained from a shape particle filtering is also combined into the final segmentation for the epi- and endocardium with an additional coupling term for the two contours [13].

Unal et al. offer a novel learning-based method for segmentation of the inner and outer arterial walls from intravascular ultrasound images. Their method is based on shape and image intensity priors along with global image measurements. They also incorporate an anatomic feature detection step to account for calcifications and branch openings in IVUS pullbacks to further improve the quality of the arterial wall extraction, which will later be utilized in plaque analysis during the assessment of the atherosclerosis disease [14].

Van Soest et al. address the problem of correcting for image degradation due to nonuniform rotation distortion encountered in imaging modalities that use a mechanically rotated endoscopic probe. To that end, they present a new method to align individual lines in a sequence of images that accurately captures slow intraframe variations in rotational velocity of the probe [15].

Danilouchkine et al. present a method for quantitative assessment of the amount of vessel wall tissue motion due to catheter rotation. Their method is based on the scale-space optical flow algorithm with a feature-based weighting scheme and it shows reliability in prediction of catheter rotational motion up to $4^{\circ}[16]$.

Roy et al. present a method for indexing echocardiograms for better content management. Specifically, the authors propose a hierarchical state-based model for representing an echo video, where the semantics of each video segment are captured using the characteristics of the objects depicted [17].

In summary, the papers from this special section provide the reader with representative samples of important advances in computational methods for intravascular and intracardiac image analysis. The major challenges for the future involve advanced computational modeling of vasculature and cardiovascular disease supported by imaging, fusion, and analysis of multimodality imaging, as well as innovative image-guided interventional 
techniques for disease management and treatment. While better hardware is essential for these tasks, cutting-edge algorithms implemented on faster and more powerful computers will further improve the processing and interpretation of the imagery. Thus, we believe that the development of newer and more robust computational schemes for medical image processing is crucial in order to get the maximum benefit out of the latest imaging devices being introduced by the major original equipment manufacturers.

\section{GOZDE UNAL, Guest Editor}

Faculty of Engineering and Natural Sciences

Sabanci University

34956 Istanbul, Turkey

\section{Greg SlabAugh, Guest Editor \\ Siemens Corporate Research \\ Princeton, NJ 08540 USA}

\section{IOANNIS A. KAKADIARIS, Guest Editor}

Computer Science, ECE, Biomedical Engineering

Houston, TX 77204 USA

\section{Allen TANnENBAUM, Guest Editor \\ Schools of Electrical and Computer \\ and Biomedical Engineering \\ Gerogia Institute of Technology, \\ Atlanta GA 30332 USA}

\section{REFERENCES}

[1] T. Kume, T. Akasaka, T. Kawamoto, N. Watanabe, E. Toyota, Y. Neishi, R. Sukmawan, Y. Sadahira, and K. Yoshida, "Assessment of coronary arterial plaque by optical coherence tomography" Am.J. Cardiol., vol. 97, no. 8, pp. 1172-1175, 2006.
[2] G. Pasterkamp, E. Falk, H. Woutman, and C. Borst, "Techniques characterizing the coronary atherosclerotic plaque: Influence on clinical decision making?," J. Am. Coll. Cardiol., vol. 36, no. 1, pp. 13-21, 2000.

[3] C. L. deKorte, A. F. Van Der Steen, E. I. Cepedes et al., "Characterization of plaque components and vulnerability with intravascular ultrasound elastography," Phys. Med. Biol., vol. 45, pp. 1465-1475, 2000.

[4] A. Wahle, G. P. M. Prause, S. C. DeJong, and M. Sonka, "Geometrically correct 3-D reconstruction of intravascular ultrasound images by fusion with biplane angiography - methods and validation," IEEE Trans. Med. Imag., vol. 18, no. 8, pp. 686-699, Aug. 1999.

[5] A. Wahle, G. P. M. Prause, C. Von Birgelen, R. Erbel, and M. Sonka, "Fusion of angiography and intravascular ultrasound in vivo: Establishing the absolute 3-D frame orientation," IEEE Trans. Biomed. Eng., vol. 46, no. 10 , pp. $1176-1180$, Oct. 1999.

[6] D. Gil, A. Hernandez, O. Rodriguez, J. Mauri, and P. Radeva, "Statistical strategy for anisotropic adventitia modelling in IVUS," IEEE Trans. Med. Imag., vol. 25, no. 6, pp. 768-778, Jun. 2006.

[7] Proceedings of the 1st International Workshop on Computer Vision for Intravascular and Intracardiac Imaging, in Medical Image Computing and Computer Assisted Intervention (MICCAI) (2006, Oct 6). [Online]. Available: http://vpa.sabanciuniv.edu/sites/cvii2006/

[8] R. A. Baldewsing, M. G. Danilouchkine, F. Mastik, J. A. Schaar, P. W. Serruys, and A. F. W. van der Steen, "An inverse method for imaging the local elasticity of atherosclerotic coronary plaques." (in this Special Issue)

[9] R. L. Maurice, J. Fromageau, M.-H. Roy Cardinal, M. Doyley, E. Demuinck, J. Robb, and G. Cloutier, "Characterization of Atherosclerotic Plaques and Mural Thrombi with Intravascular Ultrasound Elastography: A Potential Method Evaluated in an Aortic Rabbit Model and a Human Coronary Artery." (in this Special Issue)

[10] S. M. O'Malley, J. Granada, S. Carlier, M. Naghavi, and I. A. Kakadiaris, "Image-based gating of intravascular ultrasound pullback sequences," in Proc. IEEE Int. Conf. (ICASSP 2007), vol. 1, no. 15-20, pp. I-433-I-436, Apr.

[11] M. Jourdain, J. Meunier, J. Sequeira, J.-M. Boï, and J.-C. Tardif, "Robust 3D IVUS transducer tracking using single-plane cineangiography." (in this Special Issue)

[12] A. Katouzian, B. Baseri, E. E. Konofagou, and S. G. Carlier, "Challenges in atherosclerotic plaque characterization with intravascular ultrasound (IVUS): From data collection to classification." (in this Special Issue)

[13] J. Folkesson, E. Samset, R. Y. Kwong, and C.-F. Westin, "Unifying statistical classification and geodesic active regions for segmentation of cardiac MRI." (in this Special Issue)

[14] G. Unal, S. Bucher, S. G. Carlier, G. Slabaugh, T. Fang, and K. Tanaka, "Shape-driven segmentation of the arterial wall from intravascular ultrasound images." (in this Special Issue)

[15] G. van Soest, J. G. Bosch, and A. F. W. van der Steen, "Azimuthal registration of image sequences affected by non-uniform rotation distortion."

[16] G. Danilouchkine, F. Mastik, and A. F. W. van der Steen, "Accuracy in prediction of catheter rotation in IVUS with feature-based optical flow-a phantom study." (in this Special Issue)

[17] A. Roy, S. Sural, J. Mukherjee, and A. K. Majumdar, "State-based modeling and object extraction from echocardiagram video." (in this Special Issue)

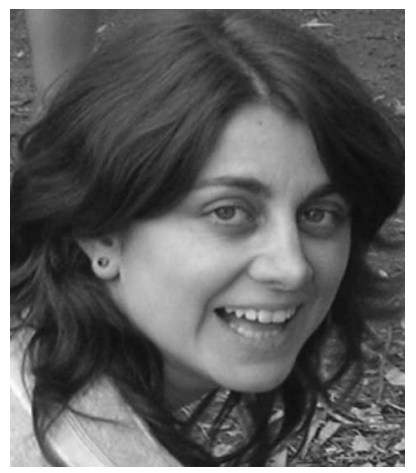

Gozde Unal (S'96-M'02-SM'08) received the Ph.D. degree in electrical engineering from North Carolina State University, Raleigh, in August 2002.

She was a Postdoctoral Fellow at the Georgia Institute of Technology, Atlanta, in 2002-2003. From Fall 2003 to 2007, she was a Research Scientist at Siemens Corporate Research, Princeton, NJ. She joined Sabanci University, Istanbul, Turkey, in Fall 2007, where she is currently an Assistant Professor. Her research interests include variational techniques with connection to information theory and probability theory, applications to various computer vision problems such as stereoscopic camera calibration, 2-D/3-D image segmentation and registration, filtering and enhancement, and stochastic particle systems. Her current research is focused on medical image analysis, segmentation, registration, and shape analysis techniques with applications to clinically relevant problems in magnetic resonance, computed tomography, Ultrasound, and intravascular images.

Dr. Unal was the General Chair of the 1st International Workshop on Computer Vision for Intravascular Imaging held in conjunction with the Medical Image Computing and Computer-Assisted Intervention (MICCAI) in 2006. 


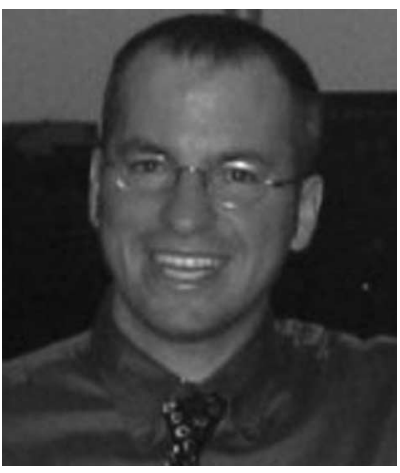

Greg Slabaugh (S'98-M'02) received the Ph.D. degree in electrical engineering from Georgia Institute of Technology, Atlanta, in 2002.

He is currently a Project Manager at Siemens Corporate Research, Princeton, NJ.

Dr. Slabaugh is an Associate Editor of the IEEE SignAl Processing MAGAZINE. He coorganized the International Workshop on Computer Vision for Intravascular Imaging held in conjunction with the Medical Image Computing and Computer-Assisted Intervention (MICCAI) 2006. His main research interests are in computer vision, image processing, and computer graphics. His current research interests include medical image processing, 3-D reconstruction from multiple images, image and surface registration, segmentation, geometric modeling, adaptive filtering, partial differential equations, radial basis functions, deformable surfaces, and level set methods.

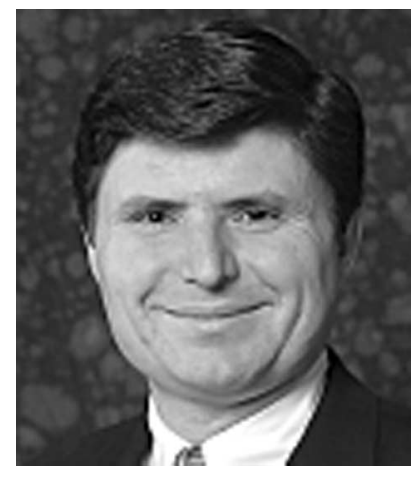

Ioannis A. Kakadiaris received the Bachelor's degree in physics from the University of Athens, Athens, Greece, the Master's degree in computer science from Northeastern University, Boston, MA, and the Ph.D. degree from the University of Pennsylvania, Philadelphia.

He is currently an Eckhard Pfeiffer Professor of Computer Science, Electrical and Computer Engineering, and Biomedical Engineering at the University of Houston, Houston, TX, where he founded the Computational Biomedicine Laboratory. His current research interests include cardiovascular informatics, biomedical image analysis, computer vision, and pattern recognition. $\mathrm{He}$ is the Co-Inventor of in vivo vasa vasorum detection using differential imaging.

Prof. Kakadiaris is the recipient of numerous awards, including the National Science Foundation (NSF) Early Career Development Award, the Schlumberger Technical Foundation Award, the UH Computer Science Research Excellence Award, the UH Enron Teaching Excellence Award, and the James Muller Vulnerable Plague Young Investigator Prize. His research has been featured on National Public Radio, KPRC NBC News, KTRH ABC News, and KHOU CBS News. Dr. Kakadiaris served at a Program Co-Chair for the 1st International Workshop on Computer Vision for Intravascular Imaging held in conjunction with the Medical Image Computing and Computer-Assisted Intervention (MICCAI) in 2006.

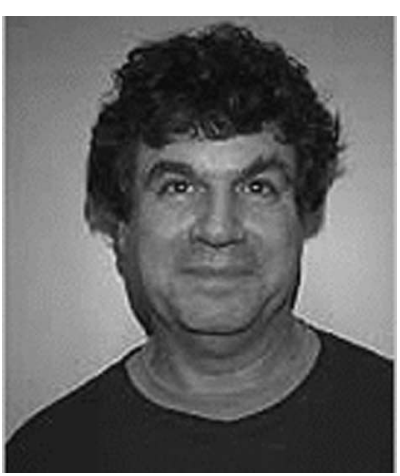

Allen Tannenbaum (M'93) received the Ph.D. degree in mathematics from Harvard University, Cambridge, MA, in 1976.

He has held faculty positions at the Weizmann Institute of Science, Ben-Gurion University of the Negev, the Technion (Israel Institute of Technology), and the University of Minnesota. In August 1999, he joined the Department of Electrical and Computer Engineering, Georgia Institute of Technology (GT), Atlanta, where he set up the Laboratory for Computational Computer Vision and is currently the Julian Hightower Professor of Engineering. He is also associated with the GT/Emory Department of Biomedical Engineering, Atlanta, as a Professor. He has played a leading role in developing new mathematical techniques for various engineering problems in systems and control, vision, signal processing, and cryptography. He is the author or coauthor of more than 360 publications including five research texts on systems and control.

Prof. Tannenbaum is the recipient of numerous awards, and has given plenary talks at a number of conferences in engineering and mathematics. His current research interests include medical imaging, control, and computer vision. 